\title{
Identification of kinetic triplets by results of derivatographic analysis
}

\author{
Ivan S. Bondarchuk ${ }^{1,4}$, Sergei S. Bondarchuk ${ }^{2,3}$, and Boris V. Borisov ${ }^{4, *}$ \\ ${ }^{1}$ National Research Tomsk State University, 634050 Tomsk, Russia \\ ${ }^{2}$ Tomsk State Pedagogical University, 634061 Tomsk, Russia \\ ${ }^{3}$ Institute for Problems of Chemical and Energetic Technologies SB RAS, 659322 Biisk, Russia \\ ${ }^{4}$ National Research Tomsk Polytechnic University, 634050 Tomsk, Russia
}

\begin{abstract}
The method for identification of the triplet of kinetic parameters of a heterogeneous reaction using the data of the derivatographic analysis is proposed. This method is characterized by high accuracy and relative simplicity and it can be effectively realized using MS Excel software.
\end{abstract}

Metal nanopowder is one of the most prospective high-energy materials that is widely used in many spheres $[1,2]$. This explains long and sustained interest in studying the features of metal nanopowder, in particular the kinetics of oxidation [3]. The proceeding heterogeneous reaction can be in full measure characterized by the so-called "kinetic triplet" - the frequency factor $\mathrm{A}$, the activation energy $E_{a}$ and the mathematical description of the reaction mecha$\operatorname{nism} f(\alpha)[4,5,6]$.

To identify these three parameters, the data of the derivatographic experiment conducted in the non-isothermal mode are used, when the change in the degree of transformation of the material $\alpha$ over time $t$ is described by the differential equation

$$
\frac{d \alpha}{d t}=A \cdot \exp \left(-\frac{E_{a}}{R T}\right) \cdot f(\alpha), \quad R=8.3144621 \frac{\mathrm{J}}{\mathrm{mol} \cdot \mathrm{K}} .
$$

Under the temperature-linear heating where the rate of temperature increase $\mathrm{T}$ with a given parameter $\beta=d T / d t$ and a specific mechanism $f(\alpha)=(1-\alpha)^{n}$, the kinetic equation (1) can be represented as

$$
\frac{d \alpha}{d t}=\frac{A}{\beta} \cdot \exp \left(-\frac{E_{a}}{R T}\right) \cdot(1-\alpha)^{n},
$$

where $n$ - order (index) of reaction.

Equation (2) has no analytical solution and numerical methods or approximate analytical ones are used to determine the kinetic parameters.

The aim of this paper is to represent a quantitative result analysis of the derivatographic study of oxidation on the basis of a new approach to calculating the kinetic parameters of a

\footnotetext{
*Corresponding author: bvborisov@tpu.ru
} 
heterogeneous reaction, as well as to obtain relationships for the estimation of these parameters.

Identification of the kinetic parameters is performed with measured values $\left\{T_{i}, \alpha_{i}\right\}$ ( $i=$ $0,1, \ldots, N$ and is determined by minimizing the coefficient of variation calculated in accordance with (2) the values of the frequency factor $A_{i}$

$$
A_{i}=\beta \frac{\int_{\alpha_{0}}^{\alpha_{i}} \frac{d \alpha}{(1-\alpha)^{n}}}{\int_{T_{0}}^{T_{i}} \exp \left(-\frac{E_{a}}{R T}\right) d T}, i=1, \ldots, N
$$

The integral in the numerator of equation (3) has a following quadrature

$$
\int_{\alpha_{0}}^{\alpha} \frac{d \alpha}{(1-\alpha)^{n}}=- \begin{cases}\ln \left[\left(1-\alpha_{0}\right) /(1-\alpha)\right], & \text { if } n=1 \\ {\left[\left(1-\alpha_{0}\right)^{1-n}-(1-\alpha)^{1-n}\right] /(1-n),} & \text { if } n \neq 1\end{cases}
$$

The denominator integral of the Arrhenius shape is calculated by approximation [7]

$$
\int_{0}^{T} \exp \left(-\frac{E_{a}}{R T}\right) d T \approx \frac{R T^{2}}{E_{a}} \cdot \frac{\frac{E_{a}}{R T}+0.25403 \ln \left(\frac{E_{a}}{R T}\right)+0.36665}{\frac{E_{a}}{R T}+0.24598 \ln \left(\frac{E_{a}}{R T}\right)+2.41457} \exp \left(-\frac{E_{a}}{R T}\right),
$$

that provides calculation accuracy with a relative error approximately estimated to $10-4 \%$.

Fig. 1 shows a solution of the example from the monograph [8] by using the method described above; discrepancy between parameters $\mathrm{n}$ and $E_{a}$ is less than $0.01 \%$.

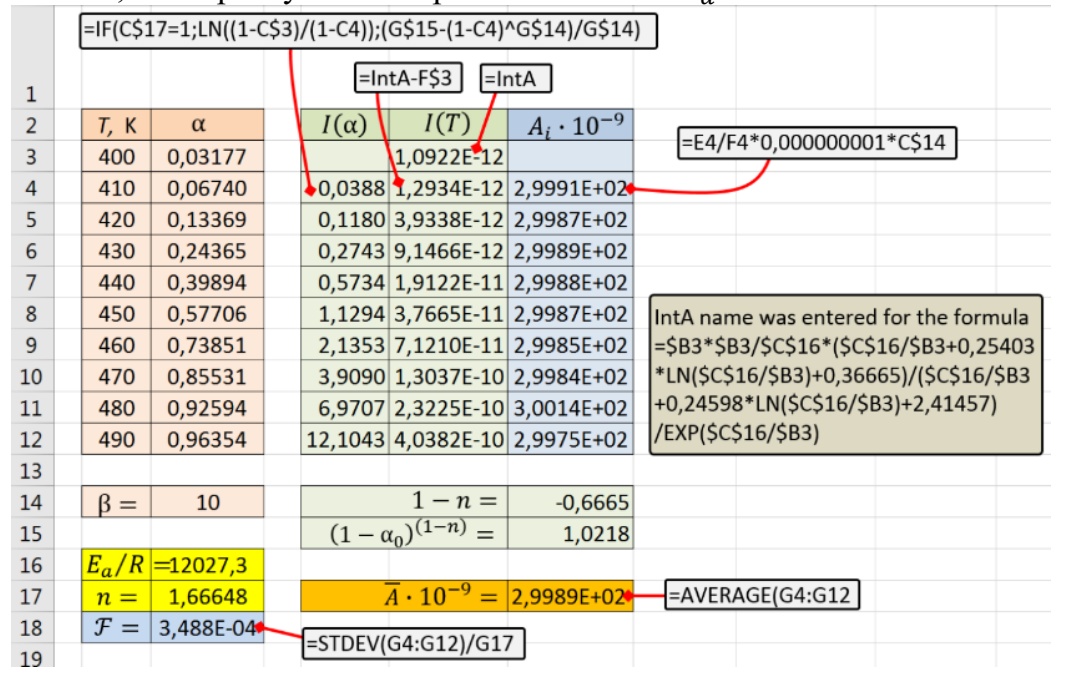

Fig. 1. Screenshot showing the solution of identification of kinetic parameters.

The minimization of the spread of the calculated values (on fig. 1 this functional - the objective function - is represented by $F$ ) was conducted using Solver tool in the mode (for the given case) of the nonlinear generalized decreasing gradient method [9] for the scalable values of $E_{a} / R$ and $n$.

To estimate the kinetic parameters of the reactions $A, E_{a}$ and $n$ the degree of conversion $\alpha$ as a function of temperature in some approximation can be described (approximated) by equation. 


$$
\alpha=\frac{1}{1+\exp \left[\frac{-2 \pi\left(T-T_{0}\right)}{\Delta T}\right]}, \quad T \in\left[T_{0}-\frac{\Delta T}{2} ; T_{0}+\frac{\Delta T}{2}\right],
$$

that depends on two parameters: distinctive temperature T_0 of the highest reaction rate, that defines half reaction of a matter $\alpha=0.55$ and temperature range $\Delta T$ of an area $\left(1+\mathrm{e}^{\pi}\right)^{-1} \leq \alpha \leq\left(1+\mathrm{e}^{-\pi}\right)^{-1}$ of aprox. $90 \%$ of material transformation (fig. 2 ).

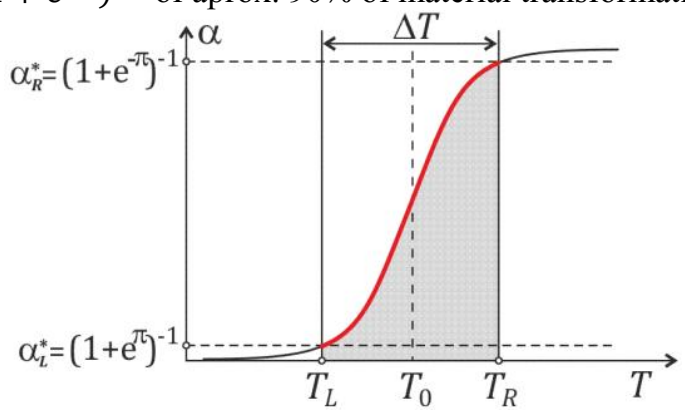

Fig. 2. Qualitative character of the derivatogramm

Using the approximation differential (4) and the kinetic equation of the process (2) for the representative values of the temperature $T_{L}=T_{0}-\Delta T / 2, T=T_{0}$ and $T_{R}=T_{0}+\Delta T / 2$ we can determine the main parameters of the reaction:

$$
\begin{gathered}
n=\frac{2 \cdot \ln \left(\operatorname{ch} \frac{\pi}{2}\right)}{\ln \left(\operatorname{ch} \frac{\pi}{2}\right)+\frac{\pi}{4} \frac{\Delta T}{T_{0}}} \approx \frac{2}{1+0.8537 \frac{\Delta T}{T_{0}}}, \\
E_{a}=\pi n R \frac{T_{0}^{2}-(\Delta T / 2)^{2}}{\Delta T}, \\
A=2^{n-1} \frac{\pi \beta}{\Delta T} \cdot \exp \left(\frac{E_{a}}{R T_{0}}\right) .
\end{gathered}
$$

Output parameters $T_{0}$ and $\Delta T$ for the ratios (5) are defined by temperatures $T_{L}$ and $T_{R}$ using for example a method of proportional parts of initial relation $\left\{T_{i} ; \alpha_{i}\right\}$.

The analysis of the parameters of the same example [4] according to the ratios (5) results in the following deviations from the precise solution: $3 \%$ deviation from the reaction order $n$ and $2.9 \%$ deviation from the activation energy $E_{a}$.

Thus, in this paper the method for identification of the triplet of kinetic parameters of a heterogeneous reaction using the data of the derivatographic analysis is proposed. This method is characterized by high ac-curacy and relative simplicity and it can be effectively realized using MS Excel software, which is available on almost every personal computer whether at a laboratory, school or at home. In addition, the method of approximate estimation of kinetic parameters on the basis of dependence approximation between the degree of material transformation and the temperature of the logistic curve is proposed.

This work was supported in part by the Russian Foundation for Basic Research (Project No. 16-3860031 mol_a_dk). 


\section{References}

1. S. Vorozhtsov, I. Zhukov, A. Vorozhtsov, A. Zhukov, D. Eskin, A. Kvetinskaya, Adv. Mater. Sci. Eng. 2015, 718207 (2015)

2. I. Zhukov, S. Vorozhtsov, V. Promakhov, I. Bondarchuk, A. Zhukov, A. Vorozhtsov, JPCS 652 (2015)

3. A. B. Vorozhtsov, M. Lerner, N. Rodkevich, H. Nie, A. Abraham, M. Schoenitz, E. L. Dreizin, Thermochim. Acta. 636, 48 (2016)

4. I. S. Bondarchuk, I. A. Zhukov, V. V. Promakhov, S. A. Vorozhtsov, Multifunctional Chemical Materials and Technologies: Abstracts International Scientific Conference (TSU, Tomsk, 2015)

5. J. Zaripov, B. Borisov, and S. Bondarchuk, EPJ Web Conf. 76, 01032 (2014)

6. I. S. Bondarchuk, I. A. Zhukov, V. V. Promakhov, PFSD-2018 (TSU, Tomsk, 2015)

7. C. Junmeng, H. Fang, W. Yi, Y. Fusheng, Chem Eng J. 124, 18 (2006)

8. J. E. House, Principles of Chemical Kinetics (Academic Press, Cambridge, 2007)

9. I. S. Bondarchuk, I. A. Kurzina, S. S. Bondarchuk, Higher Education Today 9, 22 (2014) 